Präv Gesundheitsf 2012 · 7:3-4

DOI 10.1007/s11553-011-0327-9

Online publiziert: 7. Februar 2012

(c) Springer-Verlag 2012

B. Wallmann

Zentrum für Gesundheit, Deutsche Sporthochschule, Köln

\title{
Gesundheitskompetenz - Aufbauen auf einem starken Fundament
}

und berichten über die Ergebnisse einer Befragung der Mitgliedsorganisationen der Bundesvereinigung von Prävention und Gesundheitsförderung. Sie zeigen, dass die Qualitätsentwicklungsmaßnahmen gegenwärtig noch uneinheitlich und wenig formalisiert sind, so dass keine allgemeinen Aussagen zur Verbreitung und Anwendung von Qualitätsmaßnahmen getroffen werden können. Zukünftig gebührt diesem komplexen Feld unbedingt eine weitere umfassende Bearbeitung.

Die Erfahrung bisheriger Praxis hat gelehrt, dass der Zugangsweg zur Zielgruppe das „A“ und „O“ der Gesundheitsförderung darstellt. Dass „Attraktivität“ auf gesellschaftlicher Ebene einen großen Stellenwert einnimmt, nutzen M. Axt-Gadermann und N. Fisching und untersuchen mit Hilfe einer Literaturanalyse, inwieweit ein propagiertes Schönheitsbild aufgrund eines gesunden Lebensstils den Zugang zur Gesundheitsförderung ebnen kann. Sie sehen darin großes Potential und schlagen vor, diesen Ansatz in $\mathrm{Zu}$ kunft insbesondere für schwer zugängliche Gruppen zu untersuchen.

Dem Tourismus als ein noch recht neues Setting der Gesundheitsförderung widmen sich Cassens et al. in ihrem Beitrag. Sie kommen zu dem Schluss, dass dieses Feld stärker in die Curricula der Hochschulen einbezogen werden sollte und dass hier großer Forschungsbedarf entstehen wird, denn gerade in der Urlaubszeit lassen sich gesundheitsbezogene Themen oft leichter platzieren.
An innovativen Trainingsmethoden mangelt es in der heutigen Zeit sicherlich nicht, doch müssen diese evaluiert sein bevor sie eine breite Anwendung finden. Die Praxis der Prävention und Therapie sollte aktuell und dynamisch bleiben, finden G. Seeber und C. Zalpour, die mögliche Auswirkungen des Slacklinings auf die Gleichgewichtsfähigkeit von Senioren untersuchen und durchaus erfolgreiche Anwendungsmöglichkeiten finden.

Unter dem Begriff der Transparenz von Gesundheitsinformationen entwickeln Bruder et al. ein Modell einer Ernährungsampel für ein Cateringunternehmen, welches dem Klientel die Nährstoffgehalte der angebotenen Mittagsmenüs demonstriert. Aus der praktischen Umsetzung haben die Autoren gelernt, dass eine Ernährungsampel sich nicht pauschal einführen lässt, sondern dass diese immer an die spezifischen Bedingungen des Klientels und die sich daraus ergebenen „Referenzwerte" geknüpft sind.

Ein immer mehr in den Forschungsfokus gelangtes Setting umfasst die Arbeitswelt. Initiativen wie die Betriebliche Gesundheitsförderung sind hier von hohem Belang und zeigen sich in den zahlreichen Berichten dieses Heftes. Eine spannende Diversität zeigt sich zwischen den unterschiedlich untersuchten Arbeitsfeldern.

Maier et al. beschäftigen sich mit dem Bereich der Psychohygiene beim Pflegepersonal in der geriatrischen Palliativpflege. Hohe psychische Belastungen, insbesondere des nicht-pflegerische Personals werden berichtet. Somit scheint es von Be- 
deutung zu sein sich nicht nur der besseren Versorgung pflegebedürftiger Personen zu widmen, sondern es muss gleichsam ein Fokus auf das Personal in Pflegeeinrichtungen gerichtet werden.

Auf Seiten der Ärzteschaft wird der Ruf nach einer gendersensiblen „worklife-balance“ lauter, die gendersensible Arbeits(zeit)modelle implizieren. Dies ist das Ergebnis von Miksch et al., die in ihrem Beitrag anhand einer Umfrage unter der nachrückenden Ärztegeneration relevante Unterschiede zwischen Frauen und Männern hinsichtlich der Ansprüche der „work-life-balance“ dokumentieren konnten.

In diesem Zusammenhang schließt sich auch der folgende Beitrag nahtlos an, der sich dem immer wachsenden Stellenwert von Lebensqualität im betrieblichen Kontext widmet. Physisch und psychisch gesunde, leistungsfähige sowie leistungsbereite Mitarbeiter sind dabei für jeden Arbeitgeber eine Voraussetzung, um im heutigen Wettbewerb bestehen zu können. Wilke et al. finden in einer Querschnittserhebung heraus, dass die körperliche Aktivität einen positiven Einfluss auf die subjektiv empfundene Gesundheit hat. Eine nachhaltige Bindung an Bewegung kann somit entscheidend für die Betriebliche Gesundheitsförderung sein.

Bartsch et al. zeigen auf, dass die Berufsgruppe der Polizeibeamten ausgeprägten Stressbelastungen unterliegen, die sich nicht selten in psychischen Erkrankungen niederschlagen. Die Autoren kommen zu dem Schluss, dass die Belastungen insbesondere durch die Minimierung von administrativen und organisationsspezifischen Faktoren entgegengewirkt werden kann.

Der Bildungssektor, insbesondere an den weiterführenden Schulen, wies in den letzten Jahren steigende Arbeitsunfähigkeitstage auf. Dabei stellen Störungen wie Stress und Burnout die meistgenannten Gründe dar. Sowohl ältere als auch jüngere Lehrkräfte würden von flächendeckenden Gesundheitsförderungsmaßnahmen profitieren, wie man in der Analyse von Griebler et al. nachlesen kann.

Und auch im Setting Hochschule ist alltagsintegrierte Bewegungsförderung nicht nur sinnvoll, sondern erfolgreich durchzuführen dokumentieren König et al. Dabei ist die die Nachfrage bezüglich einer Verankerung von körperlicher Aktivität in den Hochschulalltag über alle Statusgruppen hinweg gewollt. Grundlegende Voraussetzung bleibt aber, dass Angebote niedrigschwellig, umfassend und nachhaltig sind.

Die vorliegenden Beiträge dieses Heftes zeigen die Forschungsergebnisse in Lebenswelten und Settings sowie neue Handlungsansätze in der Gesundheitsförderung und Prävention auf. Die Kompetenzvermittlung jedes Einzelnen bleibt eine der Hauptmaxime, um eindimensionale Ansätze zu verhindern und damit eine flächendeckende Gesundheitsförderung zu gewährleisten. Denn gerade der Zugang zu Gesundheitskompetenzen wird zukünftig noch viel stärker über das Gesundheitsverhalten jedes Einzelnen entscheiden und dieses prägen.

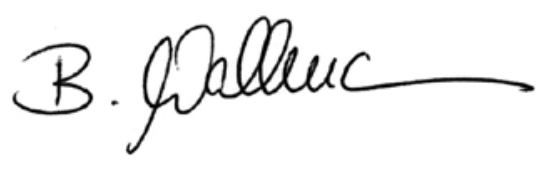

B. Wallmann

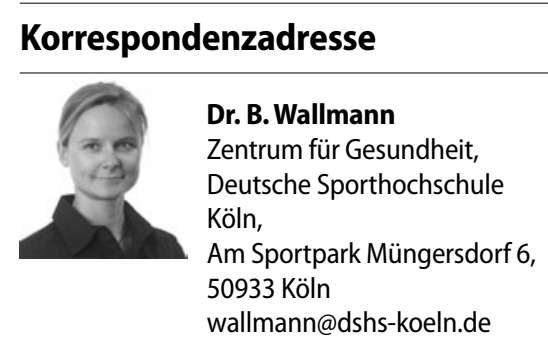

